\title{
Weighted Hardy-Sobolev inequality and global existence result of thermoelastic system on manifolds with corner-edge singularities
}

\author{
Morteza Koozehgar Kalleji ${ }^{1}$ \\ ${ }^{1}$ Arak University
}

May 6, 2020

\begin{abstract}
This article concerns with the thermoelastic corner-edge type system with singular potential function on a wedge manifold with corner singularities. First, we introduce weighted $\$$ p- $\$$ Sobolev spaces on manifolds with corner-edge singularities. Then, we prove the corner-edge type Sobolev inequality, Poincar $\$ \backslash$ acute $\{\mathrm{e}\} \$$ inequality and Hardy inequality and obtain some results about the compactness of embedding maps on the weighted corner-edge Sobolev spaces. Finally, as an application of these results, we apply the potential well theory and the Faedo-Galerkin approximations to obtain the global weak solutions for the thermoelastic corner-edge type system.
\end{abstract}

\section{Hosted file}

corner-edge singularity.pdf available at https://authorea.com/users/317348/articles/447451weighted-hardy-sobolev-inequality-and-global-existence-result-of-thermoelastic-systemon-manifolds-with-corner-edge-singularities 